•研究报告・

\title{
同域分布共享传粉者的鼠尾草属 植物的生殖隔离
}

\author{
魏宇昆 ${ }^{*}$ 黄艳波 李桂彬 \\ (上海辰山植物园, 中国科学院上海辰山植物科学研究中心, 上海 201602)
}

\begin{abstract}
摘要: 生殖隔离是生物多样性产生的重要原因之一, 不同物种间的生殖隔离形成阶段、方式和强度不完全相同。 为了揭示生殖隔离在鼠尾草属(Salvia)物种多样性产生和维持过程中的作用和特点, 本文研究了浙江天目山同域 分布的舌瓣鼠尾草(S. liguliloba)和南丹参(S. bowleyana)的开花、传粉和生殖隔离, 利用人工授粉和杂交实验检测它 们的种间遗传相容程度。结果表明, 在花的形态结构、花及花序的生长、着生方式与数量特征上, 2 种鼠尾草属植 物均差异显著 $(P<0.05)$ 。2种鼠尾草属植物共享同一种有效传粉昆虫—三三条熊蜂(Bombus trifasciatus), 但通过访 花行为的显著差异和明显不同的花粉落置部位, 有效避免了异源花粉干扰。人工杂交授粉的结果表明 2 个物种间遗 传相容性较高, 互为亲本的杂交结实率分别达到 $77.8 \pm 10.7 \%$ 和 $78.7 \pm 11.2 \%$ 。我们的研究表明, 同域分布、花期重 叠且共享唯一传粉者的 2 种鼠尾草属植物, 以花部的传粉结构和传粉部位的差异, 产生了较高程度的传粉前生殖 隔离, 避免了物种间的异源花粉干扰和自然杂交, 保障各自较高的繁殖成效, 以维持自然种群的多样性和遗传结 构的稳定。
\end{abstract}

关键词：机械隔离; 遗传相容性; 物种多样性; 自然杂交; 三条熊蜂

\section{Reproductive isolation in sympatric Salvia species sharing a sole pollinator}

Yukun Wei*, Yanbo Huang, Guibin Li

Shanghai Chenshan Plant Science Research Center, Chinese Academy of Sciences; Shanghai Chenshan Botanical Garden, Shanghai 201602

Abstract: Reproductive isolation (RI) is one of the key factors for speciation and diversity maintenance, however, there are differences in formation stage, means, and strength of RI for different species. To assess the effects and the significance of reproductive isolation in Salvia species, we compared flowering, pollination, and reproduction characteristics of Salvia liguliloba and S. bowleyana, which grow sympatrically and have overlapping flowering periods in the Tianmu Mountain, Zhejiang. Furthermore, artificial hybridization between the two species were conducted to estimate and understand their genetic compatibility and reproductive isolation. Results indicated that the two Salvia species considerably differed in their flower morphological structures, inflorescence organization, and the number of flowers produced per inflorescence. Bombus trifasciatus is the sole pollinator for these two species during the overlapping flowering period. However, different visitation behavior and floral structure $(P<0.05)$ leads to obviously different pollination patterns between the two species. Pollen is deposited on distinctly separate areas of the pollinator's body in a manner precluding contact with any heterospecific Salvia stigmata. Under natural condition, the two species are equally successful in reproduction (natural seed set $>87 \%$ ). Artificial pollination and hybridization experiments between these two species showed that they have a higher genetic compatibility. The cross seed sets are $77.8 \pm 10.7 \%$ and $78.7 \pm 11.2 \%$ when the two species are reciprocally cross parents. Our research suggests that for the two Salvia species lacking genetic incompatibility systems and relying on pollinators for outcrossing, there are differences in floral structures and pollen placement sites. The mechanical isolation (a form of pre-pollination RI) prevents heterospecific pollen interference and natural hybridization of the two

收稿日期: 2017-05-27; 接受日期: 2017-06-21

基金项目: 上海市绿化和市容管理局资助项目(G162408, G172410)

* 通讯作者 Author for correspondence. E-mail: ykwei@sibs.ac.cn 
different species that grow sympatrically and have overlapping flowering periods, and also maintains species diversity and the constancy of species heredity.

Key words: mechanical isolation; genetic compatibility; species diversity; natural hybridization; Bombus trifasciatus

生殖隔离是物种形成和多样性产生的重要机 制(Widmer et al, 2009; Baack et al, 2015), 也是避免 物种自然杂交不利因素的重要途径。根据植物繁殖 过程中隔离机制产生的阶段，可将生殖隔离分为传 粉前(pre-pollination)和传粉后(post-pollination)。传 粉前隔离主要包括物种间的生态位分化(如地理隔 离)、表型隔离(如物候花期不同、花传粉结构的差 异等)和传粉者特化(物种间花粉干扰减少); 传粉后 隔离包括不同来源花粉与柱头的竞争、杂交不亲 和、杂交种子产量、活力以及 $\mathrm{F}_{1}$ 代幼苗适应能力降 低等(Melo et al, 2014; Baack et al, 2015; Ma et al, 2016a)。不同物种的生殖隔离机制有所不同。对于 同域分布物种而言, 花结构表型相似的物种对传粉 者的竞争在被子植物花结构多样性分化中具有重 要作用(Kipling \& Warren, 2013)。

鼠尾草属(Salvia)的物种丰富度很高, 分布较 为广泛(魏宇昆等, 2015)。前期的研究表明鼠尾草属 植物存在普遍的同域物种分布现象, 这可能与其花 部结构和繁育系统的多样性密切相关(黄艳波等, 2014，2015), 但是对鼠尾草属植物传粉和生殖隔离 的研究有限。同域分布的多个鼠尾草属物种生殖隔 离主要通过生态位分化、物候差异和传粉者特化等 几种方式, 物种间的遗传不相容性非常低(ClaßenBockhoff et al, 2004), 这说明传粉后隔离的程度很 弱, 物种多样性的维持可能更多地取决于花的表型 变化与传粉者, 以及不同类型的传粉前隔离等综合 的生殖隔离机制。然而, 目前我们对鼠尾草属植物 的传粉前隔离现象仍然了解不多, 对其隔离机制以 及传粉者与植物的相互关系、生殖隔离对鼠尾草属 物种多样性的维持作用认识不够。另一方面, 鼠尾 草属植物普遍的同域物种分布现象(Claßen-Bockhoff et al, 2004)很可能会导致较高的自然杂交概率 (Epling, 1947; Meyn \& Emboden, 1987), 种间杂交 和生殖隔离如何维持动态平衡以保持物种多样性 的稳定，它们与物种形成和演化关系等问题的阐明， 都需要对物种繁育系统和传粉生态学的深入了解 和认识(Claßen-Bockhoff et al, 2003)。基于此, 本研
究以同域分布的 2 种鼠尾草属植物舌瓣鼠尾草 (Salvia liguliloba) 和南丹参(S. bowleyana) 为研究对 象, 研究比较2种植物的开花、传粉特征和繁殖; 通 过人工杂交实验了解 2 个物种的遗传相容程度和杂 交结实率，以期能够认识 2 个物种的生殖隔离方式 和强度, 为阐明鼠尾草属的物种多样性形成和维持 机制奠定基础。

\section{1 材料与方法}

\section{1 样地选择和研究对象}

样地位于浙江省天目山国家级自然保护区内 $\left(30^{\circ} 23^{\prime} 29.60^{\prime \prime} \mathrm{N}, 119^{\circ} 29^{\prime} 01.77^{\prime \prime} \mathrm{E}\right.$, 海拔677 m), 气候 类型属北亚热带季风性气候, 植物区系的温带、亚 热带东亚区系成分特征显著(杨逢春, 1992)。选择舌 瓣鼠尾草和南丹参的天然混生群落为研究样地。将 分布在样地内的植株移植到相邻的 2 个样方中, 组成 个体数量相等的 2 个居群(各 50 株), 以利于在相同的 环境条件下观察记录传粉昆虫的传粉行为和访花特 征。研究的舌瓣鼠尾草为1-2年生草本植物, 天目山 -黄山区系特有物种, 株高30-70 cm, 植株单一或 偶有分枝, 以基生叶为主，2-4对茎生叶; 南丹参为 多年生草本植物，广布于长江以南地区，株高 50-70 cm, 秋冬季以基生叶为主, 花果期主要为茎 生叶, 分枝较多。2种植物在保护区内较多见, 常于 林下、路边等生境形成优势种群; 物候特征基本相 同，花期为5月中旬至6月中旬，果期从5月下旬开 始, 6月初陆续进入成熟期。

\section{2 花部结构测量统计}

植物的花结构形态、组成和开花方式对传粉者 行为有显著的影响, 因此我们对 2 种鼠尾草的花部 结构形态、组成和开花方式做了测量和统计。随机 选取样方内 6 个不同植株上完全开放的 12 朵小花, 用电子游标卡尺对各花部结构性状如花冠长、花冠 宽、花冠高、冠筒长、冠口高、冠筒口高、冠筒口 宽、花丝长、药隔长、雌荵长等10个指标进行测量, 具体方法参考黄艳波等(2015)。鼠尾草属的花序由2 个聚伞花序为基本单位，组成2至多花的轮伞花序， 
再由多个轮伞花序组成顶生假总状花序, 或组成2 个以上分枝的顶生假圆雉花序。属内不同鼠尾草的 花序多样性表现在组成聚伞花序基本单位的小花 数量、轮伞花序数和花序总数等。我们对 2 种鼠尾草 花器官的不同生长方式、数量和开花特征进行统计, 包括单株花序总数、单花序侧生分枝数、单一分枝 的轮伞花序数、轮伞花序小花总数、轮伞花序同时 开放小花数、单株总花数和单株总开花数。

\section{3 传粉和杂交实验}

利用SONY数码摄录机(HDR-CX510E)记录样 方内 2 种鼠尾草属植物单株的昆虫访花情况。在天 气晴好的情况下, 从 8:00-18:00进行不间断录像, 记录访花昆虫和访花时间, 鉴定昆虫的种类, 统计 昆虫的数量和访花次数, 重复录制 3 天。统计分析有 效传粉昆虫对 2 种植物的访花相对频次 (relative frequency, $F$ )、单花访花时间、活跃度(activity rate, $A R)$ 和访花频率。活跃度以单只昆虫每分钟的访问 花朵数量表示, 代表了此种昆虫对某一种植物花的 偏好程度和传粉潜力; 访花频率用指数 $I_{\mathrm{VR}}=F \times$ $A R$ 表示, 表示单株植物的昆虫访花频率, 该指数同 时考虑了昆虫的活跃度及其访花相对频次(黄艳波 等, 2015)。

本研究对2种鼠尾草分别进行异交、自交和种 间杂交的人工授粉实验, 对比分析 3 种处理下的植 株结实率, 了解 2 个物种的繁育系统, 验证生殖隔
离的方式和程度。对舌瓣鼠尾草和南丹参各15株分 别进行异株异花授粉和同株异花授粉实验，杂交实 验以舌瓣鼠尾草和南丹参交互作为父本和母本进 行人工授粉, 2 种杂交组合各重复 5 株。作为父本的 鼠尾草取花药刚开裂时的具较高活力的花粉, 母本 的材料作去雄处理, 待雌苾成熟后进行人工授粉, 对 于选取进行授粉实验的花序, 在开花前至结实的过 程中作套袋处理，避免传粉昆虫干扰。结实率计算待 果实完全成熟后进行, 根据整株植物的总花朵数和 小坚果总数计算结实率, 方法如下: 结实率 $(\%)=$ 小 坚果总数/总胚珠数 $\times 100$ 。最后采用SPSS 21 和Excel 2016对各组数据进行统计学和差异显著性分析。

\section{2 结果}

\section{1 花部结构和开花特征}

对花部结构的测量结果表明, 2种鼠尾草形态 特征差异显著(图1)。舌瓣鼠尾草花冠颜色由白色过 渡至浅粉色，药隔上臂较其他部分的颜色更深; 而 南丹参花冠整体浅黄色具紫色斑点, 其下唇和雄荵 下臂顶端为显著的紫红色。舌瓣鼠尾草整体形态呈 细长筒形, 花冠水平直伸, 冠口部略大, 高与宽近 乎相等, 浅黄色花药紧贴上唇; 而南丹参冠筒细窄 至冠口显著开张, 其整体形态呈镰刀状, 花冠高远 大于宽, 花药完全内藏于两侧紧密折合的上唇之 内。从花形态结构测定指标可以看出, 2种鼠尾草除
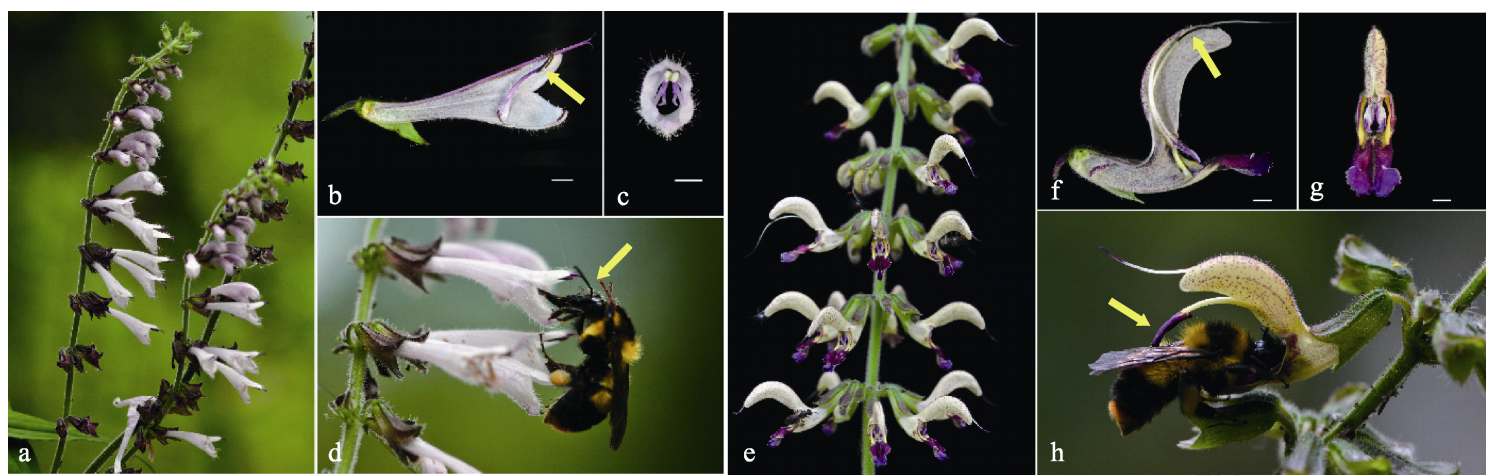

图1 舌瓣鼠尾草和南丹参的花序、花结构及昆虫传粉特征(图中标尺均为 $2 \mathbf{m m}$ )。(a)舌瓣鼠尾草花序; (b)舌瓣鼠尾草花冠纵 切(箭头所示为雄荵花药); (c)舌瓣鼠尾草花正面观; (d)三条熊蜂访问舌瓣鼠尾草及其传粉过程(箭头所示为传粉部位); (e)南丹 参花序; (f)南丹参花冠纵切(箭头所示为雄荵花药); (g) 南丹参花正面观; (h)三条熊蜂访问南丹参及其传粉过程(箭头所示为传 粉部位)。

Fig. 1 Comparison of inflorescences, flower structures, and insect pollinations of Salvia liguliloba and S. bowleyana $($ Bar $=2 \mathrm{~mm})$. (a) Inflorescences of S. liguliloba; (b) Flower lateral dissection of S. liguliloba (the arrow indicates anther); (c) A front view of $S$. liguliloba flower; (d) Bombus trifasciatus is visiting flowers and pollinating S. liguliloba (the arrow indicates pollen placement sites on bumblebee); (e) Inflorescences of S. bowleyana; (f) Flower lateral dissection of S. bowleyana (the arrow indicates anther); (g) A front view of S. bowleyana flower; (h) Bombus trifasciatus is visiting flowers and pollinating S. bowleyana (the arrow indicates pollen placement sites on bumblebee). 
表1 舌瓣鼠尾草与南丹参的花部结构比较(平均值 \pm 标准差)

Table 1 Comparison of flower structures between Salvia liguliloba and S. bowleyana (mean \pm SD)

\begin{tabular}{|c|c|c|c|c|c|c|c|c|c|c|}
\hline & $\begin{array}{l}\text { 花冠长 } \\
\text { Corolla } \\
\text { length }\end{array}$ & $\begin{array}{l}\text { 花冠宽 } \\
\text { Corolla } \\
\text { width }\end{array}$ & $\begin{array}{l}\text { 花冠高 } \\
\text { Corolla } \\
\text { height }\end{array}$ & $\begin{array}{l}\text { 冠筒长 } \\
\text { Tube } \\
\text { length }\end{array}$ & $\begin{array}{l}\text { 冠口高 } \\
\text { Entrance } \\
\text { height }\end{array}$ & $\begin{array}{l}\text { 冠筒口高 } \\
\text { Tube entra- } \\
\text { nce height }\end{array}$ & $\begin{array}{l}\text { 冠筒口宽 } \\
\text { Tube entra- } \\
\text { nce width }\end{array}$ & $\begin{array}{l}\text { 花丝长 } \\
\text { Filament } \\
\text { length }\end{array}$ & $\begin{array}{l}\text { 药隔长 } \\
\text { Connective } \\
\text { length }\end{array}$ & $\begin{array}{l}\text { 雌荵长 } \\
\text { Pistil } \\
\text { length }\end{array}$ \\
\hline $\begin{array}{l}\text { 舌瓣鼠尾草 } \\
\text { S. liguliloba }\end{array}$ & $23.7 \pm 0.74^{\mathrm{a}}$ & $5.31 \pm 0.34^{\mathrm{a}}$ & $7.30 \pm 0.67^{b}$ & $19.5 \pm 0.8^{\mathrm{a}}$ & $4.87 \pm 0.61^{b}$ & $4.87 \pm 0.61^{\mathrm{a}}$ & $4.41 \pm 0.52^{\mathrm{a}}$ & $2.39 \pm 0.14^{\mathrm{b}}$ & $5.19 \pm 0.33^{b}$ & $23.7 \pm 0.43^{b}$ \\
\hline $\begin{array}{l}\text { 南丹参 } \\
\text { S. bowleyana }\end{array}$ & $22.8 \pm 1.10^{\mathrm{b}}$ & $4.47 \pm 0.26^{\mathrm{b}}$ & $16.6 \pm 0.94^{\mathrm{a}}$ & $12.4 \pm 0.76^{\mathrm{b}}$ & $11.2 \pm 0.80^{\mathrm{a}}$ & $4.47 \pm 0.28^{\mathrm{a}}$ & $3.21 \pm 0.23^{b}$ & $4.30 \pm 0.26^{\mathrm{a}}$ & $14.3 \pm 0.68^{\mathrm{a}}$ & $26.1 \pm 1.85^{\mathrm{a}}$ \\
\hline$P$ & 0.0407 & 0.000 & 0.000 & 0.000 & 0.000 & 0.0585 & 0.000 & 0.000 & 0.000 & 0.0011 \\
\hline
\end{tabular}

统计分析使用 $t$-检验，同一列不同字母代表差异显著 $(P<0.05)$ 。 The different letters for the same column indicate significant difference at the level of $P<0.05$ by $t$-test.

表2 舌瓣鼠尾草和南丹参花及花序数量特征(平均值 \pm 标准差)

Table 2 Comparisons of flowering and inflorescence between Salvia liguliloba and S. bowleyana (mean \pm SD)

\begin{tabular}{|c|c|c|c|c|c|c|c|}
\hline & $\begin{array}{l}\text { 单株花序总数 } \\
\text { No. of infloresce- } \\
\text { nce in individual } \\
\text { plant }\end{array}$ & $\begin{array}{l}\text { 假圆雉花序 } \\
\text { 分枝数 } \\
\text { No. of branch } \\
\text { in a false panicle }\end{array}$ & $\begin{array}{l}\text { 假总状花序的 } \\
\text { 轮伞花序数 } \\
\text { No. of verticillaster } \\
\text { in a false raceme }\end{array}$ & $\begin{array}{l}\text { 轮伞花序小 } \\
\text { 花总数 } \\
\text { r No. of flower in } \\
\text { a verticillaster }\end{array}$ & $\begin{array}{l}\text { 轮伞花序同时 } \\
\text { 开放小花数 } \\
\text { No. of flowering } \\
\text { in a verticillaster }\end{array}$ & $\begin{array}{l}\text { 单株总花数 } \\
\text { Total no. of } \\
\text { flower in } \\
\text { individual plant }\end{array}$ & $\begin{array}{l}\text { 单株总开花数 } \\
\text { Total no. of } \\
\text { flowering in } \\
\text { individual plant }\end{array}$ \\
\hline $\begin{array}{l}\text { 舌瓣鼠尾草 } \\
\text { S. liguliloba }\end{array}$ & $2.0 \pm 2.1^{\mathrm{a}}(\mathrm{n}=35)$ & $2.0 \pm 1.9^{\mathrm{b}}(\mathrm{n}=39)$ & $9.3 \pm 2.4^{\mathrm{a}}(\mathrm{n}=83)$ & $5.6 \pm 1.1^{\mathrm{b}}(\mathrm{n}=93)$ & $1.4 \pm 1.7^{\mathrm{b}}(\mathrm{n}=234)$ & $262 \pm 299^{\mathrm{b}}(\mathrm{n}=26)$ & $21 \pm 30^{\mathrm{a}}(\mathrm{n}=36)$ \\
\hline $\begin{array}{l}\text { 南丹参 } \\
\text { S. bowleyana }\end{array}$ & $2.2 \pm 0.45^{\mathrm{a}}(\mathrm{n}=5)$ & $5.8 \pm 3.0^{\mathrm{a}}(\mathrm{n}=11)$ & $9.5 \pm 1.9^{\mathrm{a}}(\mathrm{n}=40)$ & $9.8 \pm 1.9^{\mathrm{a}}(\mathrm{n}=160)$ & $1.8 \pm 1.0^{\mathrm{a}}(\mathrm{n}=160)$ & $891 \pm 472^{\mathrm{a}}(\mathrm{n}=5)$ & $48 \pm 56^{a}(n=16)$ \\
\hline$P$ & 0.681 & 0.0020 & 0.7166 & 0.000 & 0.0053 & 0.0005 & 0.089 \\
\hline
\end{tabular}

统计分析使用 $t$-检验，同一列不同字母代表差异显著 $(P<0.05)$ 。Different letters for the same column indicate significant difference at the level of $P<0.05$ by $t$-test.

了冠筒口高外, 其他各项指标均差异显著 $(P<0.05)$ (表1), 舌瓣鼠尾草的花冠长、花冠宽、冠筒长、冠 筒口宽显著大于南丹参, 而南丹参的花冠高、冠口 高、花丝长、药隔长和雌荵长则显著大于舌瓣鼠尾 草, 其中两者的药隔长度差异极显著 $(P<0.01)$ 。

本研究的2种鼠尾草在花部着生和排列方式, 以及单株的花朵和开花数量上都有所不同(表2)。统 计结果表明, 南丹参的假圆雉花序分枝数、轮伞花 序小花总数、轮伞花序同时开放小花数和单株总花 数均明显多于舌瓣鼠尾草 $(P<0.05)$ 。而单株花序总 数和假总状花序的轮伞花序数 2 种植物并没有显著 差异, 尽管南丹参的单株总开花数要多于舌瓣鼠尾 草, 但两者差异并不显著。总体而言在单株水平上, 南丹参的花器官组成较舌瓣鼠尾草更复杂, 花朵数 量更多, 可能会影响传粉昆虫对 2 种植物的传粉行为。

\subsection{2 种鼠尾草的传粉机制比较}

植物的传粉机制与其花形态结构和传粉者的 行为密切相关。我们的观察结果表明, 样地中的舌 瓣鼠尾草与南丹参共享一种有效传粉昆虫即三条 熊蜂(Bombus trifasciatus)来完成各自的授粉。从传 粉过程来看, 南丹参为经典的背部杜杆传粉机制,
传粉昆虫三条熊蜂落至花冠下唇后进入冠筒吸食 花蜜, 在此过程中推动雄荵药隔下臂向内运动的同 时, 药隔上臂的花药向下运动, 雄荵花丝与药隔连 接部分具有一个可往复运动的“轴”, 该结构使得传 粉昆虫进出冠筒时, 药隔能够稳定、准确地将花药 的花粉涂抹于三条熊蜂的背部, 当昆虫退出花冠后 雄萝又能恢复到最初的位置。南丹参精确传粉的关 键在于其结构特化的雄荵和与其相适应的花冠形 态, 如显著伸长的雄荵上臂, 可运动的“轴” 以及两 侧折合呈镰刀状的上唇, 保证了传粉时雄荵以杜杆 方式运动, 非传粉时上唇对花药的保护; 2 个雄荵药 隔下臂的退化花药联合形成的衍生组织和较窄的 花冠口宽度, 使得传粉昆虫进入冠筒时必须推动下 臂才能够获取花蜜, 保证了传粉的专一性和稳定 性。

舌瓣鼠尾草则采用了完全不同的传粉模式, 表 现在传粉昆虫进入花部的方式和传粉部位与南丹 参完全不同, 而这些均缘于舌瓣鼠尾草特有的雄荵 结构和相应的花冠形态。舌瓣鼠尾草花部整体较小, 上下唇均较短且无明显区别, 相应的雄荵花丝和药 隔也明显比南丹参短。三条熊蜂取食花蜜时无法落 
表3 三条熊蜂对2种鼠尾草的访花行为比较(平均值 \pm 标准差)

Table 3 Comparisons of flower visiting behavior of Bombus trifasciatus between Salvia liguliloba and S. bowleyana (mean \pm SD)

\begin{tabular}{lllll}
\hline & $\begin{array}{l}\text { 相对频次 } \\
\text { Relative frequency }\end{array}$ & $\begin{array}{l}\text { 单花访问时间 } \\
\text { Visit time per flower }(\mathrm{s})\end{array}$ & $\begin{array}{l}\text { 活跃度 } \\
\text { Activity rate }\end{array}$ & $\begin{array}{l}\text { 访花频率 } \\
\text { Visitation rate }\end{array}$ \\
\hline 舌瓣鼠尾草 S. liguliloba & $0.14 \pm 0.017^{\mathrm{b}}(n=3)$ & $2.01 \pm 0.85^{\mathrm{a}}(n=30)$ & $9.71 \pm 3.15^{\mathrm{b}}(n=7)$ & $1.36 \pm 0.17^{\mathrm{b}}(n=3)$ \\
南丹参 S. bowleyana & $0.86 \pm 0.017^{\mathrm{a}}(n=3)$ & $1.80 \pm 0.63^{\mathrm{a}}(n=30)$ & $16.2 \pm 3.4^{\mathrm{a}}(n=52)$ & $13.9 \pm 0.28^{\mathrm{a}}(n=3)$ \\
$P$ & 0.000 & 0.2758 & 0.000 & 0.000
\end{tabular}

统计分析使用 $t$-检验，同一列不同字母代表差异显著 $(P<0.05)$ 。The different letters for the same column indicate significant difference at the level of $P<0.05$ by $t$-test.
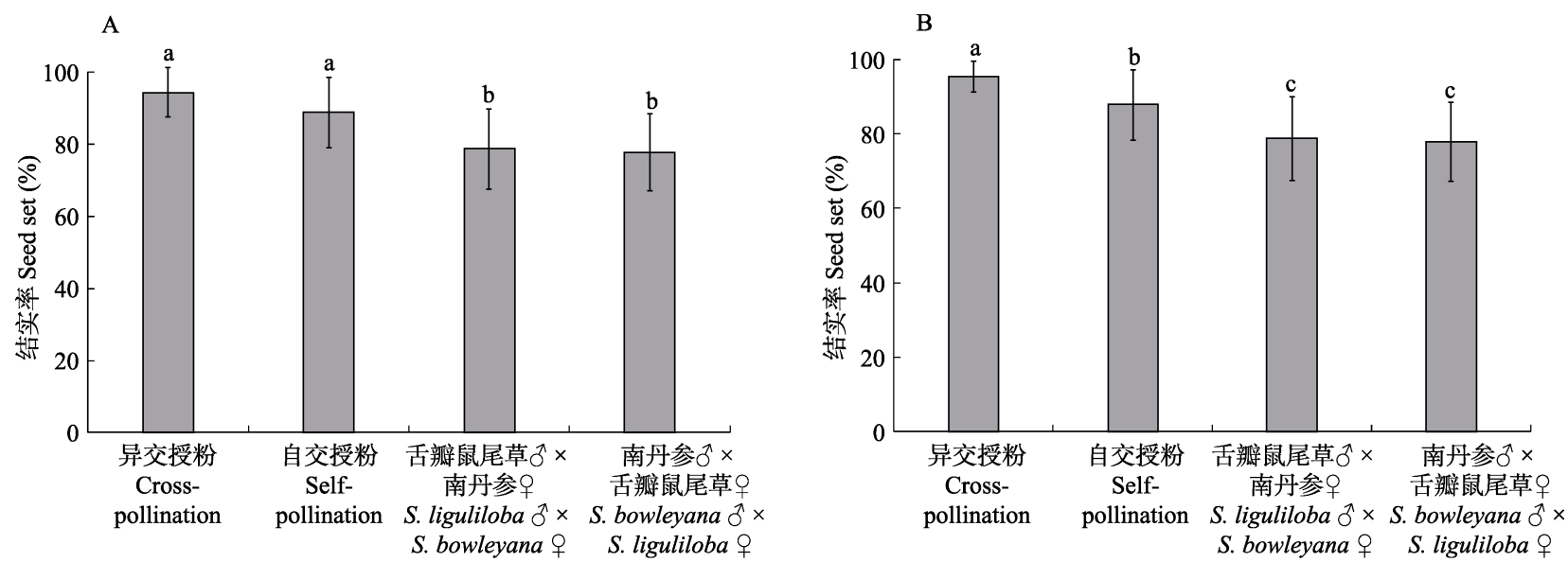

图2 2种鼠尾草的种内异交、自交和种间杂交结实率比较(平均值 \pm 标准差)。(A)舌瓣鼠尾草异交、自交以及与南丹参杂交 的结实率多重比较结果; (B)南丹参异交、自交以及与舌瓣鼠尾草杂交的结实率多重比较结果。统计分析采用 $t$-检验, 不同字 母代表差异显著 $(P<0.05)$ 。

Fig. 2 Comparisons of cross-pollination, self-pollination, and hybridization of Salvia liguliloba and S. bowleyana. (A) and (B) show S. liguliloba and S. bowleyana, respectively. Different letters indicate significant difference at the level of $P<0.05$ by $t$-test.

至花冠下唇而是直接接近冠筒, 额部抵住花冠上唇 吸食花蜜，此时位于上唇内侧的雄芯花药接触熊蜂 的额部, 当其离开时即带走花粉。在整个传粉过程 中, 雄芯的位置没有发生改变, 花丝和药隔连接部 位没有类似南丹参的可保证雄荵运动的“轴”结构。 舌瓣鼠尾草通过较小的冠口、较长的花冠筒和紧贴 上唇的雄荵花药，同样保证了三条熊蜂传粉过程的 专一、稳定、精确和有效。

访花行为的研究结果表明, 三条熊蜂对南丹参 的访花相对频次 $(P<0.001)$ 、活跃度 $(P<0.001)$ 和访 花频率 $(P<0.001)$ 均显著高于舌瓣鼠尾草(表3), 传 粉昆虫对舌瓣鼠尾草的单花访问时间稍长于南丹 参, 但差异不显著 $(P=0.2758)$ 。结果表明, 在单株 水平上，三条熊蜂对南丹参的访花频率更高。

\section{3 繁育系统和种间杂交}

从舌瓣鼠尾草和南丹参各自异交和自交授粉 实验可以看出, 2 种植物的结实率都很高, 但它们的 异交和自交结实率之间的差异有所不同(图2)。舌瓣
鼠尾草异交和自交的结实率没有显著差异 $(P=$ 0.0996), 异交结实率(94.4 \pm 6.8\%)略高于自交结实 率(88.9 \pm 9.7\%); 南丹参的异交结实率(95.3 $\pm 4.1 \%)$ 也同样高于自交 $(87.8 \pm 9.5 \%)$, 但两者具有显著性 差异 $(P<0.05)$ 。2 个物种间的交互杂交结果表明，舌 瓣鼠尾草和南丹参互为父母本的杂交结实率分别 为78.7 $\pm 11.2 \%$ 和 $77.8 \pm 10.7 \%$, 种间的遗传相容性 很高。但不论舌瓣鼠尾草还是南丹参, 其异交和自 交的结实率均显著高于种间的杂交结实率 $(P<0.05)$ 。

\section{3 讨论}

在天目山同域分布的舌瓣鼠尾草和南丹参具 有共同的传粉昆虫, 在自然生境中, 2 种鼠尾草之间 形成了较完备的生殖隔离，同时与传粉者均具有专 一性的花粉传递、分发和授粉机制, 表现出对传粉 者资源的充分利用。鼠尾草属具有高度适应传粉昆 虫的花形态结构, 物种共生进化程度较高, 突出表 现在花粉输出的稳定性、精确性和有效性，同时与 
传粉者形成专一程度和忠实性较高的共存关系(黄 艳波等, 2014)。传粉者特化能够促进生殖隔离和物 种多样性的产生(Baack et al, 2015)。本文和以往的 研究均表明, 以适应传粉者的植物花部结构特化进 而引发的物种多样性分化, 可能是鼠尾草属植物多 样性形成的关键因素(Claßen-Bockhoff et al, 2004; Wester \& Claßen- Bockhoff, 2007)。2种鼠尾草尽管 具有相似的花形态, 但基于雄荵结构的细微差别, 各自独特的传粉结构, 通过各异的传粉方式, 都能 够稳定、精确而有效的传递花粉, 同时利用同一传 粉者不同传粉部位的有效机械隔离, 避免发生种 间自然杂交的可能, 从而形成了一种较完备的传粉 前生殖隔离机制, 以维持同域分布物种的遗传稳定 性和物种多样性。

从2种鼠尾草的单花结构、花序组成和开花方 式的显著差异中我们可以看出, 它们对同一传粉者 三条雄蜂的吸引方式、相互作用(传粉机制)和传粉 特征均有所不同。南丹参具备的淡黄色花冠、紫红 色的下唇和雄荵下臂可能具有比舌瓣鼠尾草更强 的吸引传粉昆虫的能力, 反映在传粉者对南丹参的 访花频次显著高于舌瓣鼠尾草, 可能暗示三条熊蜂 对南丹参的黄色或较深的紫红色具有偏好。尽管南 丹参的昆虫访花频次、访花相对频率明显较舌瓣鼠 尾草更高 $(P<0.05)$, 但两者自然条件下的结实率相 当(南丹参: $87.8 \pm 11.8 \%$; 舌瓣鼠尾草: $87.2 \pm 7.6 \%$; $P=0.880)$, 说明舌瓣鼠尾草的昆虫授粉效率更高, 传粉效果更好。由此, 我们推测2种鼠尾草采用了不 同的传粉策略, 利用一种传粉者同时达到了较高的 繁殖成效。南丹参借助更多的花朵数量、对传粉者 更强的吸引力提高三条熊蜂的访花频率, 减少访问 时间, 完成异花授粉和较高的雄性适合度; 舌瓣鼠 尾草尽管单位时间访花数量较少, 但通过更高效的 单花访花过程, 或是更理想的植物与传粉者互作, 达到同样高水平的繁殖成效和雄性适合度。

鼠尾草属与传粉者的高度协同的传粉关系暗 示传粉者物种数量不应少于宿主的物种数, 我们的 野外调查和以往的报道也显示, 一种鼠尾草通常有 多种传粉者(或称传粉功能群)完成授粉过程, 2 种鼠 尾草共用一种传粉者的情况并不常见(Claßen-Bockhoff et al, 2004)。对玉凤花属(Habenaria)和凤仙花 属(Impatiens)同域共存的多物种传粉研究表明, 每 个物种既有特定传粉者, 也有相同的传粉者, 在共
享同一传粉者的情况下, 花传粉结构和传粉部位差 异综合作用才可能保证物种间的生殖隔离(Pedron et al, 2012; Ruchisansakun et al, 2016)。本文结果显 示同域分布的2种鼠尾草对传粉者资源的利用效率 很高, 是高度适应环境的结果。尽管通过花粉落置 部位的差异, 在共用一种传粉者时能够避免大多数 情况的花粉干扰, 但这并非绝对, 小概率的花粉干 扰事件可能无法避免(Huang \& Shi, 2013)。对于亲 缘关系较远的物种而言, 因其遗传不相容或花粉柱 头无法识别, 不会影响各自的繁殖成功(Huang et al, 2015), 但对于舌瓣鼠尾草和南丹参 2 个没有遗传不 相容性的物种来说, 如何尽可能降低少量的花粉干 扰值得深入研究。

舌瓣鼠尾草和南丹参的花期完全重叠, 在共享 同一种传粉昆虫的情况下, 通过机械隔离机制(传 粉部位的差异)避免了自然杂交后代的产生, 是一 种典型的传粉前隔离机制, 这种方式保证了2种鼠 尾草属植物较高的传粉专一性, 也暗示了该类群在 植物与传粉昆虫进化的程度较高。传粉者介导的不 同传粉模式能够促进花的分化及其引起的生殖隔 离, 表明其在植物多样性起源中的重要作用, 但很 少有研究能够阐明具有不同传粉模式的多个物种 的共存机制(Pauw, 2013)。对于植物生殖隔离的进化 而言, 传粉前隔离通常比传粉后隔离更为重要(Ma et al, 2016a,b)。鼠尾草属生殖隔离的产生可能源于 该属物种快速形成过程中的物种分化或局地环境 适应，但其起源机制仍不清楚，这种较完备的传粉 前机械隔离是如何产生的? 这需要更广范围的调 查取样以及对 2 种鼠尾草及其近缘物种的系统进化 和谱系地理学分析。生殖隔离可能更强烈地依赖于 植物的传粉系统而不是植物本身, 换言之, 植物生 殖隔离的进化更有可能受到其传粉昆虫的影响和 选择(Hopkins, 2013)。但同时我们看到, 2组人工杂 交组合尽管有较高的结实率, 但比 2 个鼠尾草亲本 各自的同种异花授粉结实率仍低了10-20\% (图2), 说明舌瓣鼠尾草和南丹参之间仍存在较弱的传粉 后隔离。

综上所述, 未来对2物种重叠分布区传粉昆虫 种类、传粉行为、传粉报酬、物候等传粉前隔离方 式和传粉后生殖隔离的花粉竞争能力、杂交种子活 力和 $F_{1}$ 代生存力的比较研究, 将有助于更全面地认 识鼠尾草属的生殖隔离模式。 
致谢: 感谢中国科学院上海辰山植物科学研究中心 严岳鸿研究员对文章撰写提出的中肯建议; 同时感谢 两位匿名审稿专家的宝贵意见和耐心细致的修改。

\section{参考文献}

Baack E, Melo MC, Rieseberg LH, Ortiz-Barrientos D (2015) The origins of reproductive isolation in plants. New Phytologist, 207, 968-984.

Claßen-Bockhoff R, Speck T, Tweraser E, Wester P, Thimm S, Reith M (2004) The staminal lever mechanism in Salvia L. (Lamiaceae): a key innovation for adaptive radiation? Organisms Diversity \& Evolution, 4, 189-205.

Claßen-Bockhoff R, Wester P, Tweraser E (2003) The staminal lever mechanism in Salvia L. (Lamiaceae): a review. Plant Biology, 5, 33-41.

Epling C (1947) Natural hybridization of Salvia apiana and S. mellifera. Evolution, 1, 69-78.

Hopkins R (2013) Reinforcement in plants. New Phytologist, 197, 1095-1103.

Huang S, Shi X (2013) Floral isolation in Pedicularis: how do congeners with shared pollinators minimize reproductive interference? New Phytologist, 199, 858-865.

Huang YB, Wei YK, Ge BJ, Wang Q (2014) Research progress in pollination biology of genus Salvia (Lamiaceae) and their pollination mechanisms in East Asia (China). Acta Ecologica Sinica, 34, 2282-2289. (in Chinese with English abstract) [黄艳波, 魏宇昆, 葛斌杰, 王琦 (2014) 鼠尾草属 东亚分支的传粉模式. 生态学报, 34, 2282-2289.]

Huang YB, Wei YK, Wang Q, Xiao YE, Ye XY (2015) Floral morphology and pollination mechanism of Salvia liguliloba, a narrow endemic species with degraded lever-like stamens. Chinese Journal of Plant Ecology, 39, 753-761. (in Chinese with English abstract) [黄艳波, 魏宇昆, 王琦, 肖月娥, 叶 喜阳 (2015) 舌瓣鼠尾草退化杜杆雄荵的相关花部特征 及传粉机制. 植物生态学报, 39, 753-761.]

Huang Z, Liu H, Huang S (2015) Interspecific pollen transfer between two coflowering species was minimized by bumblebee fidelity and differential pollen placement on the bumblebee body. Journal of Plant Ecology, 8, 109-115.

Kipling R, Warren J (2013) How generalists coexist: the role of floral phenotype and spatial factors in the pollination systems of two Ranunculus species. Journal of Plant Ecology, 7, 480-489.
Ma YP, Xie WJ, Sun WB, Marczewski T (2016a) Strong reproductive isolation despite occasional hybridization between a widely distributed and a narrow endemic Rhododendron species. Scientific Reports, 6, 19146.

Ma YP, Zhou RC, Milne R (2016b) Pollinator-mediated isolation may be an underestimated factor in promoting homoploid hybrid speciation. Frontiers in Plant Science, 7, 1183.

Melo MC, Grealy A, Brittain B, Walter GM, Ortiz-Barrientos D (2014) Strong extrinsic reproductive isolation between parapatric populations of an Australian groundsel. New Phytologist, 203, 323-334.

Meyn O, Emboden WA (1987) Parameters and consequences of introgression in Salvia apiana $\times$ S. mellifera (Lamiaceae). Systematic Botany, 12, 390-399.

Pauw A (2013) Can pollination niches facilitate plant coexistence? Trends in Ecology \& Evolution, 28, 30-37.

Pedron M, Buzatto CR, Singer RB, Batista JAN, Moser A (2012) Pollination biology of four sympatric species of $\mathrm{Ha}$ benaria (Orchidaceae: Orchidinae) from southern Brazil. Botanical Journal of the Linnean Society, 170, 141-156.

Ruchisansakun S, Tangtorwongsakul P, Cozien RJ, Smets EF, Niet TVD (2016) Floral specialization for different pollinators and divergent use of the same pollinator among co-occuring Impatiens species (Balsaminaceae) from Southeast Asia. Botanical Journal of the Linnean Society, 181, 651666.

Wei YK, Wang Q, Huang YB (2015) Species diversity and distribution of Salvia (Lamiaceae). Biodiversity Science, 23, 3-10. (in Chinese with English abstract) [魏宇昆, 王琦, 黄 艳波 (2015) 唇形科鼠尾草属的物种多样性与分布. 生 物多样性, 23, 3-10.]

Wester P, Claßen-Bockhoff R (2007) Floral diversity and pollen transfer mechanisms in bird-pollinated Salvia species. Annals of Botany, 100, 401-421.

Widmer A, Lexer C, Cozzolino S (2009) Evolution of reproductive isolation in plants. Heredity, 102, 31-38.

Yang FC (1992) Comprehensive Investigation Report on Natural Resource of Tianmu Mountain Nature Reserve. Zhejiang Science and Technology Press, Hangzhou. (in Chinese) [杨 逢春 (1992) 天目山自然保护区自然资源综合考察报告. 浙江科学技术出版社, 杭州.]

(责任编委: 严岳鸿 责任编辑: 时意专) 